\title{
ARTIFICIAL SATELLITES DYNAMICS: RESONANT EFFECTS
}

\author{
Jarbas Cordeiro Sampaio
}

UNESP- Univ Estadual Paulista, CEP. 12516-410 Guaratinguetá-SP, Brazil, jarbascordeiro@gmail.com

\section{Rodolpho Vilhena de Moraes}

UNIFESP- Univ Federal de São Paulo, CEP. 12231-280 São José dos Campos, SP, Brazil, rodolpho.vilhena@gmail.com

\section{Sandro da Silva Fernandes}

ITA- Inst Tecnológico de Aeronáutica, CEP. 12228-900 São José dos Campos, SP, Brazil, sandro@ita.br

\begin{abstract}
In this work, the resonance problem in the artificial satellites motion is studied. The development of the geopotential includes the zonal harmonics $J_{20}$ and $J_{40}$ and the tesseral harmonics $J_{22}$ and $J_{42}$. Through successive Mathieu transformations, the order of dynamical system is reduced and the final system is solved by numerical integration. In the simplified dynamical model, two critical angles are studied, $\phi_{2201}$ and $\phi_{4211}$. Numerical results show the time behavior of the semi-major axis and $\phi_{2}$ angle.
\end{abstract}

Keywords: Artificial Satellites. Resonance. Orbits.

\section{Introduction}

Synchronous satellites in circular or elliptical orbits have been extensively used for navigation, communication and military missions. This fact justifies the great attention that has been given in literature to the study of resonant orbits characterizing the dynamics of these satelli-tes since the 60's (Morando, 1963; Blitzer, 1963; Garfinkel, 1965a; Garfinkel, 1965b; Garfinkel, 1966; Gedeon and Dial, 1967; Gedeon et al., 1967; Gedeon, 1969; Lane, 1988; Jupp, 1969; Ely and Howell, 1996). For example, Molniya series satellites used by the old Soviet Union for communication form a constellation of approximately 110 satellites, launched since 1965, which have highly eccentric orbits with periods of 12 hours. Another example of missions that use eccentric orbits, inclined and synchronous, include satellites to investigate the solar magnetosphere, launched in the 90's (Neto, 2006).

The orbits of synchronous satellites are very complex. The tesseral harmonics of the geopotential produce multiple resonances which interact resulting significantly nonlinear motions, when compared to non-resonant orbits. It has been found that the orbital elements show relatively large oscillation amplitudes differing from neighboring trajectories, they are in fact chaotic (Ely and Howell, 1996). It should also be noted that the characteristics of several missions involving such orbits require that they are kept to a minimum fuel consumption. Geographic requirements determined by the missions and spatial maneuvers of minimum cost demand precise control of the trajectories that are subjected to significant nonlinearities during the satellite lifetime.

In this paper, the 2:1 resonance is considered; in other words, the satellite completes two revolutions while the Earth carries one.

\section{The Resonance Problem}

In this section, a simplified Hamiltonian describing the resonant problem is derived through sucessive Mathieu transformations.

Consider Eq. (1) to the Earth gravitational potential written in classical orbital elements (Osorio, 1973; Kaula, 1966)

$$
V=\frac{\mu}{2 a}+\sum_{l=2}^{\infty} \sum_{m=0}^{l} \sum_{p=0}^{l} \sum_{q=+\infty}^{-\infty} \frac{\mu}{a}\left(\frac{a_{e}}{a}\right)^{l} J_{l m} F_{l m p}(I) G_{l p q}(e) \cos \left(\phi_{l m p q}(M, \omega, \Omega, \Theta)\right)
$$

where $\mu$ is the Gaussian constant, $\mu=3.986009 \times 10^{14} \mathrm{~m}^{3} / \mathrm{s}^{2}, a, e, I, \Omega, \omega, M$ are the classical keplerian elements: 
$a$ is the semi-major axis, $e$ is the eccentricity, $I$ is the inclination of the orbit plane with the equator, $\Omega$ is the longitude of the ascending node, $\omega$ is the argument of pericentre and $M$ is the mean anomaly, respectively; $a_{e}$ is the Earth mean equatorial radius, $a_{e}=6378.140 \mathrm{~km}, J_{l m}$ is the spherical harmonic coefficient of degree $l$ and order $m, F_{l m p}(I)$ and $G_{l p q}(e)$ are Kaula's inclination and eccentricity functions, respectively. The argument $\phi_{\text {lmpq }}(M, \omega, \Omega, \Theta)$ is defined by

$$
\phi_{l m p q}(M, \omega, \Omega, \Theta)=q M+(l-2 p) \omega+m\left(\Omega-\Theta-\lambda_{l m}\right)+(l-m) \frac{\pi}{2},
$$

where $\Theta$ is the Greenwich sidereal time and $\lambda_{l m}$ is the corresponding reference longitude along the equator.

In order to describe the problem in Hamiltonian form, Delaunay canonical variables are introduced

$$
\begin{aligned}
& L=\sqrt{\mu a} \quad G=\sqrt{\mu a\left(1-e^{2}\right)} \quad H=\sqrt{\mu a\left(1-e^{2}\right)} \cos (I) \\
& l=M \quad g=\omega \quad h=\Omega .
\end{aligned}
$$

Using the canonical variables, one gets the Hamiltonian $\hat{F}$,

$$
\hat{F}=\frac{\mu^{2}}{2 L^{2}}+\sum_{l=2}^{\infty} \sum_{m=0}^{l} R_{l m}
$$

with the disturbing potential $R_{l m}$ given by

$$
R_{l m}=\sum_{p=0}^{l} \sum_{q=-\infty}^{+\infty} B_{l m p q}(L, G, H) \cos \left(\phi_{l m p q}(l, g, h, \Theta)\right)
$$

The argument $\phi_{l m p q}$ is defined by

$$
\phi_{l m p q}(l, g, h, \Theta)=q l+(l-2 p) g+m\left(h-\Theta-\lambda_{l m}\right)+(l-m) \frac{\pi}{2},
$$

and the coefficient $B_{l m p q}(L, G, H)$ by

$$
B_{l m p q}=\sum_{l=2}^{\infty} \sum_{m=0}^{l} \sum_{p=0}^{l} \sum_{q=+\infty}^{-\infty} \frac{\mu^{2}}{L^{2}}\left(\frac{\mu a_{e}}{L^{2}}\right)^{l} J_{l m} F_{l m p}(L, G, H) H_{q}^{-(l+1),(l-2 p)}(L, G)
$$

The Hamiltonian $\hat{F}$ depends explicitly on the time through the Greenwich sidereal time $\Theta$, where $\Theta=\Omega_{e} t\left(\Omega_{e}\right.$ is the Earth's angular velocity and $t$ is the time). A new variable $\theta$, conjugated to $\Theta$, is introduced in order to extend the phase space. In the extended phase space, the extended Hamiltonian $\hat{H}$ is given by

$$
\hat{H}=\hat{F}+\omega_{e} \theta
$$


For resonant orbits, it is convenient to use a new set of canonical variables. Consider the canonical transformation of variables defined by the following relations

$$
\begin{aligned}
& X=L \quad Y=G-L \quad Z=H-G \quad \Theta=\Theta \\
& x=l+g+h \quad y=g+h \quad z=h \quad \theta=\theta,
\end{aligned}
$$

where $X, Y, Z, \Theta, x, y, z, \theta$ are the modified Delaunay variables.

The new Hamiltonian $\hat{H}^{\prime}$, resulting from the canonical transformation defined by Eq. (8), is given by

$$
\hat{H}^{\prime}=\frac{\mu^{2}}{2 X^{2}}+\omega_{e} \theta+\sum_{l=2}^{\infty} \sum_{m=0}^{l} R_{l m}^{\prime}
$$

where the disturbing potential $R_{l m}^{\prime}$ is given by

$$
R_{l m}^{\prime}=\sum_{p=0}^{l} \sum_{q=-\infty}^{+\infty} B_{l m p q}^{\prime}(X, Y, Z) \cos \left(\phi_{l m p q}(x, y, z, \Theta)\right)
$$

Consider the resonance to be studied in this work; that is, the commensurability between the Earth rotation angular velocity $\Omega_{e}$ and the mean motion $n$. This commensurability can be expressed as

$$
q n-m \omega_{e} \cong 0
$$

considering $q$ and $m$ as integers. The commensurability of the resonance studied, $q / m$, is defined by $\alpha$. When this commensurability ocurrs, small divisors, associated to the tesseral harmonics, arise in the integration of the equations of motion (Lane, 1988). These terms are called resonants.

The short and long period terms can be eliminated from the Hamiltonian $\hat{H}^{\prime}$ by applying an averaging method. A reduced Hamiltonian $\hat{H}_{r}$ is obtained from the Hamiltonian $\hat{H}^{\prime}$ when only secular and resonant terms are considered. Several authors, Kaula (1966), Lima Jr (1998), Formiga and Vilhena de Moraes (2009), Vilhena de Moraes et al. (1995), Ely and Howell (1996) also use this simplified Hamiltonian to study the resonance. The reduced Hamiltonian $\hat{H}_{r}$ is given by

$$
\begin{aligned}
\hat{H}_{r}= & \frac{\mu^{2}}{2 X^{2}}+\omega_{e} \theta+\sum_{j=1}^{\infty} B_{2 j, 0, j, 0}^{\prime}(X, Y, Z)+ \\
& +\sum_{l=2}^{\infty} \sum_{m=2}^{l} \sum_{p=0}^{l} B_{l m p(\alpha m)}^{\prime}(X, Y, Z) \cos \left(\phi_{l m p(\alpha m)}(x, y, z, \Theta)\right)
\end{aligned}
$$

The canonical system of differential equations governed by $\hat{H}_{r}$ has the first integral

$$
\left(1-\frac{1}{\alpha}\right) X+Y+Z=C_{1}
$$


where $C_{1}$ is an integration constant.

Using this first integral, a Mathieu transformation

$$
(X, Y, Z, \Theta, x, y, z, \theta) \rightarrow\left(X_{1}, Y_{1}, Z_{1}, \Theta_{1}, x_{1}, y_{1}, z_{1}, \theta_{1}\right)
$$

can be defined.

This transformation is given by the following equations

$$
\begin{aligned}
& X_{1}=X \quad Z_{1}=\left(1-\frac{1}{\alpha}\right) X+Y+Z \quad \Theta_{1}=\Theta \\
& x_{1}=x-\left(1-\frac{1}{\alpha}\right) z \quad y_{1}=y-z \quad z_{1}=z \quad \theta_{1}=\theta .
\end{aligned}
$$

The subscript 1 denotes the new set of canonical variables. Note that $Z_{1}=C_{1}$ and the $z_{1}$ is an ignorable variable. So, the order of the dynamical system is reduced in one degree of freedom.

Substituting the new set of canonical variables, $X_{1}, Y_{1}, Z_{1}, \Theta_{1}, x_{1}, y_{1}, z_{1}, \theta_{1}$, in the reduced Hamiltonian given by Eq. (12), one gets the resonant Hamiltonian. The word "resonant" is used to denote the Hamiltonian $\hat{H}_{1, r s}$ which is valid for any resonance. The periodic terms in this Hamiltonian are resonant terms. The Hamiltonian $\hat{H}_{1, r s}$ is given by

$$
\begin{aligned}
\hat{H}_{1, r s}= & \frac{\mu^{2}}{2 X_{1}^{2}}+\omega_{e} \theta_{1}+\sum_{j=1}^{\infty} B_{1,2 j, 0, j, 0}\left(X_{1}, Y_{1}, Z_{1}\right)+ \\
& +\sum_{l=2}^{\infty} \sum_{m=2}^{l} \sum_{p=0}^{l} B_{1, l m p,(\alpha m)}\left(X_{1}, Y_{1}, Z_{1}\right) \cos \left(\phi_{1, l m p(\alpha m)}\left(x_{1}, y_{1}, z_{1}, \Theta_{1}\right)\right)
\end{aligned}
$$

The Hamiltonian $\hat{H}_{1, r s}$ has all resonant frequencies, relative to the commensurability $\alpha$, where the $\phi_{1, l m p(\alpha m)}$ argument is given by

$$
\phi_{1, l m p(\alpha m)}=m\left(\alpha x_{1}-\Theta_{1}\right)+(l-2 p-\alpha m) y_{1}-\phi_{1, l m p(\alpha m) 0}
$$

with

$$
\phi_{1, l m p(\alpha m) 0}=m \lambda_{l m}-(l-m) \frac{\pi}{2}
$$

The secular and resonant terms are given, respectively, by $B_{1,2 j, 0, j, 0}\left(X_{1}, Y_{1}, Z_{1}\right)$ and $B_{1, l m p(\alpha m)}\left(X_{1}, Y_{1}, Z_{1}\right)$.

Each one of the frequencies contained in $\frac{d x_{1}}{d t}, \frac{d y_{1}}{d t}, \frac{d \Theta_{1}}{d t}$ is related, through the coefficients $l, m$, to a tesseral harmonic $J_{l m}$. By varying the coefficients $l, m, p$ and keeping $q / m$ fixed, one finds, all frequencies $\frac{d \phi_{1, l m p(\alpha m)}}{d t}$ concerning to a specified resonance. 
Now, consider a single frequency among the several resonant frequencies that can be obtained from the expression

$$
\frac{\phi_{1, l m p(\alpha m)}}{d t}=m\left(\alpha \frac{d x_{1}}{d t}-\frac{d \Theta_{1}}{d t}\right)+(l-2 p-m \alpha) \frac{d y_{1}}{d t}
$$

The frequency $\dot{\phi}_{1, l m p(\alpha m)}$ for the fixed coefficients $m$ and $(l-2 p-m \alpha)$ will be the unique resonant frequency considered in the resonant Hamiltonian $\hat{H}_{1, r s}$. This frequency will be called "critical frequency".

A critical frequency can be, for example, the one that results in a smaller numerical value for $\dot{\phi}_{1, \operatorname{lm} p(\alpha m)}$, implying a effect strengthening of the resonance considered. The frequencies of the arguments $\Omega$ and $\omega$ can become more pronounced the presence of small divisors that arise in the integration of the motion equations, this depend also on the eccentricity and inclination of the orbit plane. The importance of the node and the pericentre frequencies is smaller when compared to the mean anomaly and Greenwich sidereal time, however, they also have their contribution in the resonance effect. As mentioned in preceding paragraphs, the coefficients $l, m, p$ can vary, producing different frequencies within the resonant cosines for the same resonance. These frequencies are slightly different, with small variations around the commensurability given by $\alpha \dot{x}_{1}-\dot{\Theta}_{1}$.

In the keplerian elements, the coefficient of frequency $\dot{\omega}$ assumes the values $2,0,-2$, and the coefficient of frequency $\dot{y}_{1}$ assumes the values $1,-1,-3$.

According to Eq. (16), the argument in the resonant Hamiltonian is given by

$\phi_{1, \operatorname{lm} p(\alpha m)}\left(x_{1}, y_{1}, \Theta_{1}\right)$. To determine a critical frequency, one needs to fix all the coefficients of the variable $x_{1}$, $y_{1}, \Theta_{1}$; in other words, one fixes $\alpha, m$ and $(l-2 p-m \alpha)$.

The coefficient $\alpha$ is fixed, because the type of resonance is defined; in this paper 2:1. Once the resonant angle has been chosen, the coefficients $m$ and $(l-2 p-m \alpha)$ must be fixed too.

By fixing the expression $l-2 p$ instead of the coefficients $l$ and $p$ separately, one has the possibility to vary $l$ and $p$, considering that $l-2 p$ is a certain fixed value $\mathrm{k}$. Once this critical frequency has been chosen among the possible resonant frequencies, the other periodic terms of the Hamiltonian $\hat{H_{1, r s}}$ are taken as short period terms, with frequencies different from the critical frequency.

Defining a single critical frequency, or, assuming the isolated study of each frequency, a new Hamiltonian is obtained. This new Hamiltonian is given by

$$
\begin{aligned}
\hat{H}_{1, c}= & \frac{\mu^{2}}{2 X_{1}^{2}}+\omega_{e} \theta_{1}+\sum_{j=1}^{\infty} B_{1,2 j, 0, j, 0}\left(X_{1}, Y_{1}, C_{1}\right)+ \\
& +\sum_{l=2}^{\infty} \sum_{p=0}^{l} B_{1, l m p,(\alpha m)}\left(X_{1}, Y_{1}, C_{1}\right) \cos \left(\phi_{1, l m p(\alpha m)}\left(x_{1}, y_{1}, \Theta_{1}\right)\right) .
\end{aligned}
$$

The coefficients $k=l-2 p$ and $m$ are fixed. This Hamiltonian contains secular and critical terms only. Since $k$ is a fixed value, $\hat{H}_{1, c}$ can be put in the simplified form

$$
\begin{aligned}
\hat{H}_{1, c}= & \frac{\mu^{2}}{2 X_{1}^{2}}+\omega_{e} \theta_{1}+\sum_{j=1}^{\infty} B_{1,2 j, 0, j, 0}\left(X_{1}, Y_{1}, C_{1}\right)+ \\
& +\sum_{p=S}^{\infty} B_{1, l m p,(\alpha m)}\left(X_{1}, Y_{1}, C_{1}\right) \cos \left(\phi_{1, \operatorname{lm} p(\alpha m)}\left(x_{1}, y_{1}, \Theta_{1}\right)\right)
\end{aligned}
$$

where $\frac{\mu^{2}}{2 X_{1}^{2}}+\omega_{e} \theta_{1}$ is the central part of the Hamiltonian, $\sum_{j=1}^{\infty} B_{1,2 j, 0, j, 0}\left(X_{1}, Y_{1}, C_{1}\right)$ contains only secular terms with the even zonal harmonics, and 


$$
\sum_{p=s}^{\infty} B_{1,(2 p+k) m p(\alpha m)}\left(X_{1}, Y_{1}, C_{1}\right) \cos \phi_{1,(2 p+k) m p(\alpha m)}\left(x_{1}, y_{1}, \Theta_{1}\right)
$$

represents the resonant terms that have the same critical frequency.

The canonical system of differential equations governed by the Hamiltonian $\hat{H}_{1, c}$ has the first integral

$$
(k-m \alpha) X_{1}-m \alpha Y_{1}=C_{2},
$$

where $C_{2}$ is an integration constant.

Using this integral, a new Mathieu transformation can be defined. This canonical transformation is given by the following equations

$$
\begin{aligned}
& X_{2}=X_{1} \quad Y_{2}=(k-m \alpha) X_{1}-m \alpha Y_{1} \quad \Theta_{2}=\Theta_{1} \\
& x_{2}=x_{1}+\left(\frac{k-m \alpha}{m \alpha}\right) y_{1} \quad y_{2}=-\frac{1}{m \alpha} y_{1} \quad \theta_{2}=\theta_{1} .
\end{aligned}
$$

The subscript 2 denotes the new set of canonical variables.

The Hamiltonian function is invariant with respect to this new Mathieu transformation. Thus, from Eq. (20) and (22), one gets the final Hamiltonian $\hat{H}_{2, f}$

$$
\begin{aligned}
\hat{H}_{2, f}= & \frac{\mu^{2}}{2 X_{2}^{2}}+\omega_{e} \theta_{2}+\sum_{j=1}^{\infty} B_{2,2 j, 0, j, 0}\left(X_{2}, C_{1}, C_{2}\right)+ \\
& +\sum_{p=S}^{\infty} B_{2,(2 p+k) m p(\alpha m)}\left(X_{2}, C_{1}, C_{2}\right) \cos \left(\phi_{2,(2 p+k) m p(\alpha m)}\left(x_{2}, \Theta_{2}\right)\right)
\end{aligned}
$$

where $\sum_{j=1}^{\infty} B_{2,2 j, 0, j, 0}\left(X_{2}, C_{1}, C_{2}\right)$ represents the secular terms and

$$
\sum_{p=s}^{\infty} B_{2,(2 p+k) m p(\alpha m)}\left(X_{2}, C_{1}, C_{2}\right) \cos \phi_{1,(2 p+k) m p(\alpha m)}\left(x_{2}, \Theta_{2}\right)
$$

represents the resonant terms with the same critical frequencies. Note that $Y_{2}=C_{2}$ and $y_{2}$ is an ignorable variable. The new angle $\phi_{2,(2 p+k) m p(\alpha m)}\left(x_{2}, \Theta_{2}\right)$ is given by

$$
\phi_{2,(2 p+k) m p(\alpha m)}\left(x_{2}, \Theta_{2}\right)=\phi_{2}-\phi_{2,(2 p+k) m p(\alpha m), 0}
$$

where $\phi_{2}=m\left(\alpha x_{2}-\Theta_{2}\right)$, and 


$$
\phi_{2,(2 p+k) m p(\alpha), 0}=m \lambda_{(2 p+k) m}-(2 p+k-m) \frac{\pi}{2}=\phi_{1, l m p(\alpha m) 0} .
$$

Recall that $k$ and $m$ are two fixed coefficients determined by choosing a critical resonant frequency, among the several possible resonant frequencies. The Hamiltonian $\hat{H}_{2, f}$ has all tesseral related to the chosen critical frequency.

For small eccentricities, the Hansen's coefficients can be expressed in Newcomb's polynomials and Kaula's eccentricity function (Kaula, 1966):

$$
H_{\alpha m}^{-(2 p+k+1), k}(e)=G_{(2 p+k) p(\alpha m)}(e) .
$$

Accordingly, the Hamiltonian $\hat{H}_{2, f}$ can be rewritten as

$$
\begin{aligned}
\hat{H}^{\prime}{ }_{2, f}= & \frac{\mu^{2}}{2 X_{2}^{2}}+\omega_{e} \theta_{2}+\sum_{j=1}^{\infty} B_{2,2 j, 0, j, 0}\left(X_{2}, C_{1}, C_{2}\right)+ \\
& +\sum_{p=S}^{\infty} B_{2,(2 p+k) m p(\alpha m)}\left(X_{2}, C_{1}, C_{2}\right) \cos \left(\phi_{2,(2 p+k) m p(\alpha m)}\left(x_{2}, \Theta_{2}\right)\right)
\end{aligned}
$$

with

$$
\begin{aligned}
B_{2,(2 p+k) m p(\alpha m)}\left(X_{2}, C_{1}, C_{2}\right)= & \frac{\mu^{2 p+k+2}}{X^{4 p+2 k+2}} a_{e}^{2 p+k} J_{(2 p+k) m} \times \\
& \times F_{(2 p+k) m p}\left(X_{2}, C_{1}, C_{2}\right) G_{(2 p+k) p(\alpha m)}\left(X_{2}, C_{2}\right),
\end{aligned}
$$

where the function $G_{l p q}(e)$ is the Kaula's eccentricity function. Prime denotes the simplified Hamiltonian for small eccentricities.

The dynamical system generated by Hamiltonian $\hat{H}_{2, f}^{\prime}$ is

$$
\begin{aligned}
\frac{d X_{2}}{d t}= & -m \alpha \sum_{p=S}^{\infty} B_{2,(2 p+k) m p(\alpha m)}\left(X_{2}, C_{1}, C_{2}\right) \operatorname{sen}\left(\phi_{2,(2 p+k) m p(\alpha m)}\right) \\
\frac{d \phi_{2}}{d t}= & m \alpha \frac{\mu^{2}}{X_{2}^{3}}-m \omega_{e}-m \alpha \sum_{j=1}^{\infty} \frac{\partial B_{2,2 j, 0, j, 0}\left(X_{2}, C_{1}, C_{2}\right)}{\partial X_{2}}- \\
& -m \alpha \sum_{p=S}^{\infty} \frac{\partial B_{2,(2 p+k) m p(\alpha m)}\left(X_{2}, C_{1}, C_{2}\right)}{\partial X_{2}} \cos \left(\phi_{2,(2 p+k) m p(\alpha m)}\left(x_{2}, \Theta_{2}\right)\right)
\end{aligned}
$$

The Eq. 29] and (30) represent the motion equations in a resonance of commensurability $\alpha$.

\section{Results}

Figures (1) to (8) show the time behavior of the semi-major axis and $\phi_{2}$ angle, according to the numerical integration of the motion equations, (29) and (30). The initial conditions, in the Figs. (1) to (8), for inclinations are $10^{0}$ and $55^{\circ}$, and eccentricities, 0.001 and 0.01 . The initial values of semi-major axis are around the critical semi-major axis. Note that the circulation and libration regions are differentiated by color. 


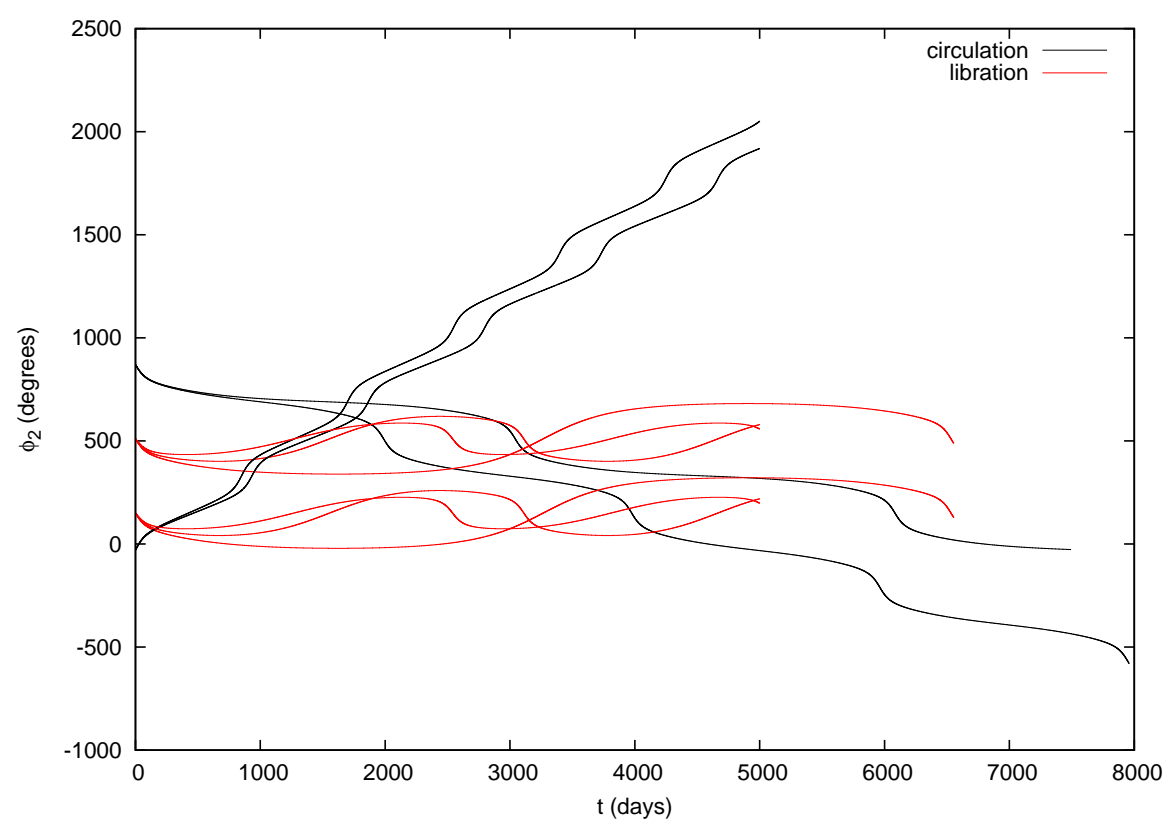

Figure 1. $\phi_{2}$ versus t, considering the tesseral harmonic $J_{22}$. The initial conditions for inclination and eccentricity are $I=10^{\circ}$ and $\mathrm{e}=0.001$, respectively

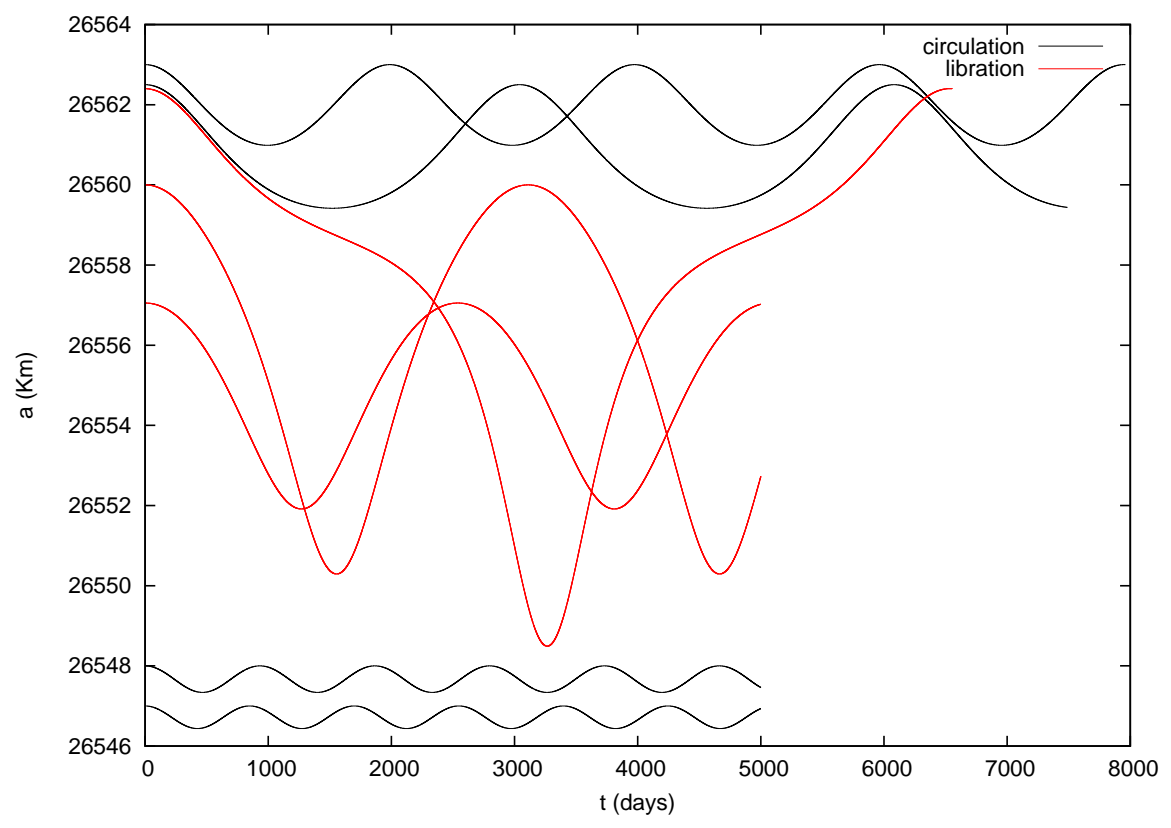

Figure 2. $a$ versus t, considering the tesseral harmonic $J_{22}$. The initial conditions for inclination and eccentricity are $I=10^{\circ}$ and $\mathrm{e}=0.001$, respectively 


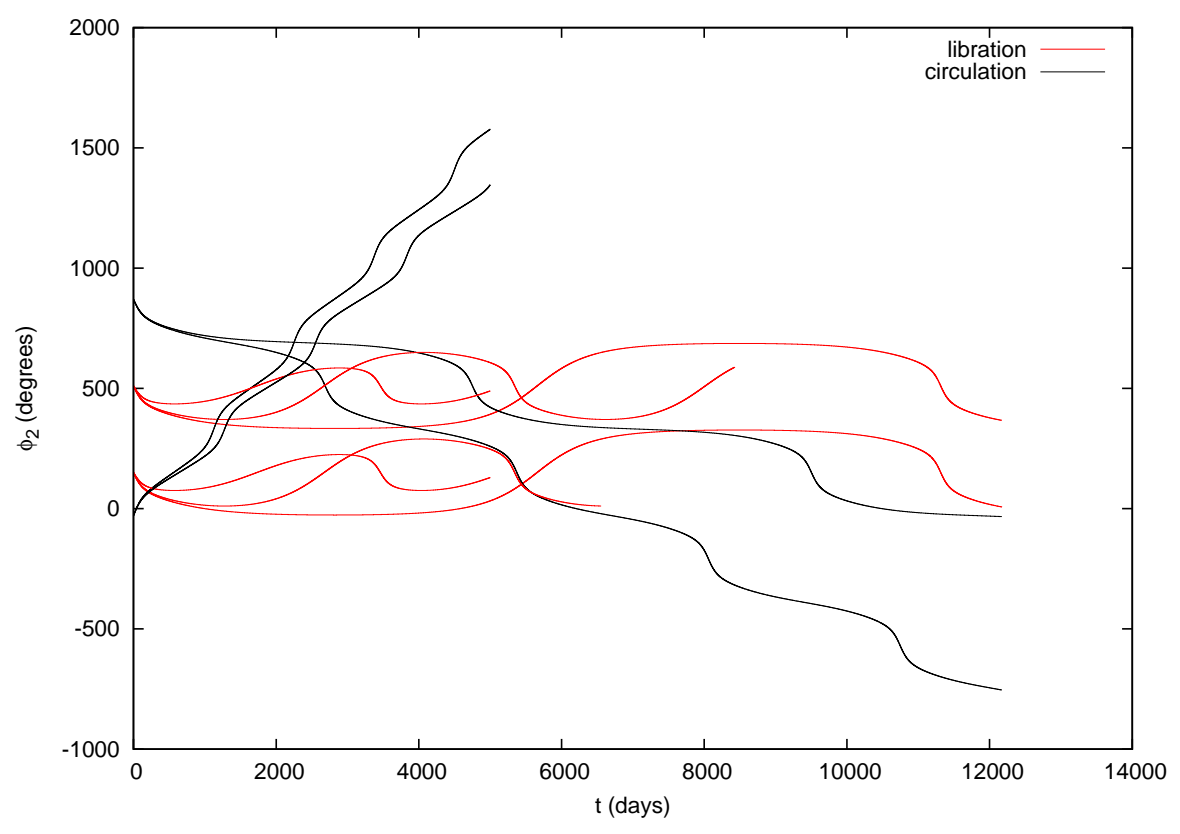

Figure 3. $\phi_{2}$ versus t, considering the tesseral harmonic $J_{22}$. The initial conditions for inclination and eccentricity are $I=55^{\circ}$ and $\mathrm{e}=0.001$, respectively

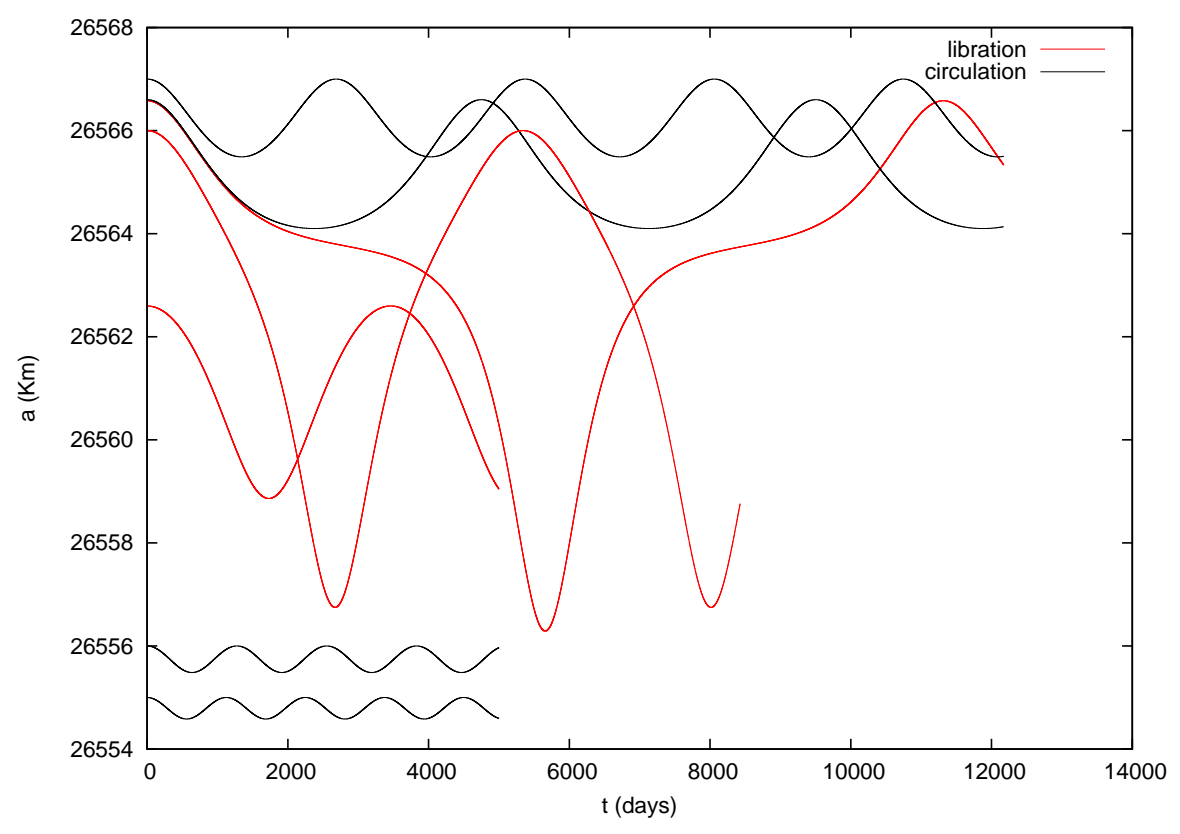

Figure 4. $a$ versus t, considering the tesseral harmonic $J_{22}$. The initial conditions for inclination and eccentricity are $I=55^{\circ}$ and $\mathrm{e}=0.001$, respectively 


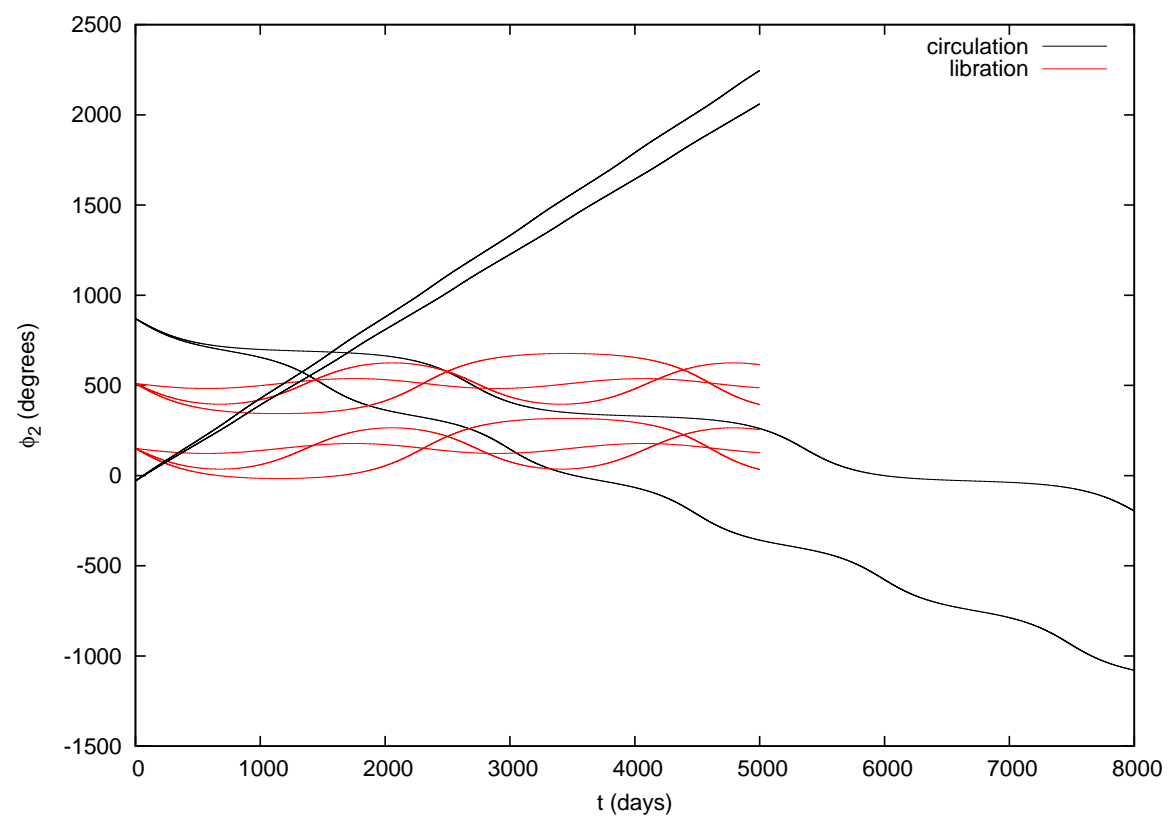

Figure 5. $\phi_{2}$ versus t, considering the tesseral harmonic $J_{22}$. The initial conditions for inclination and eccentricity are $I=10^{\circ}$ and $\mathrm{e}=0.01$, respectively

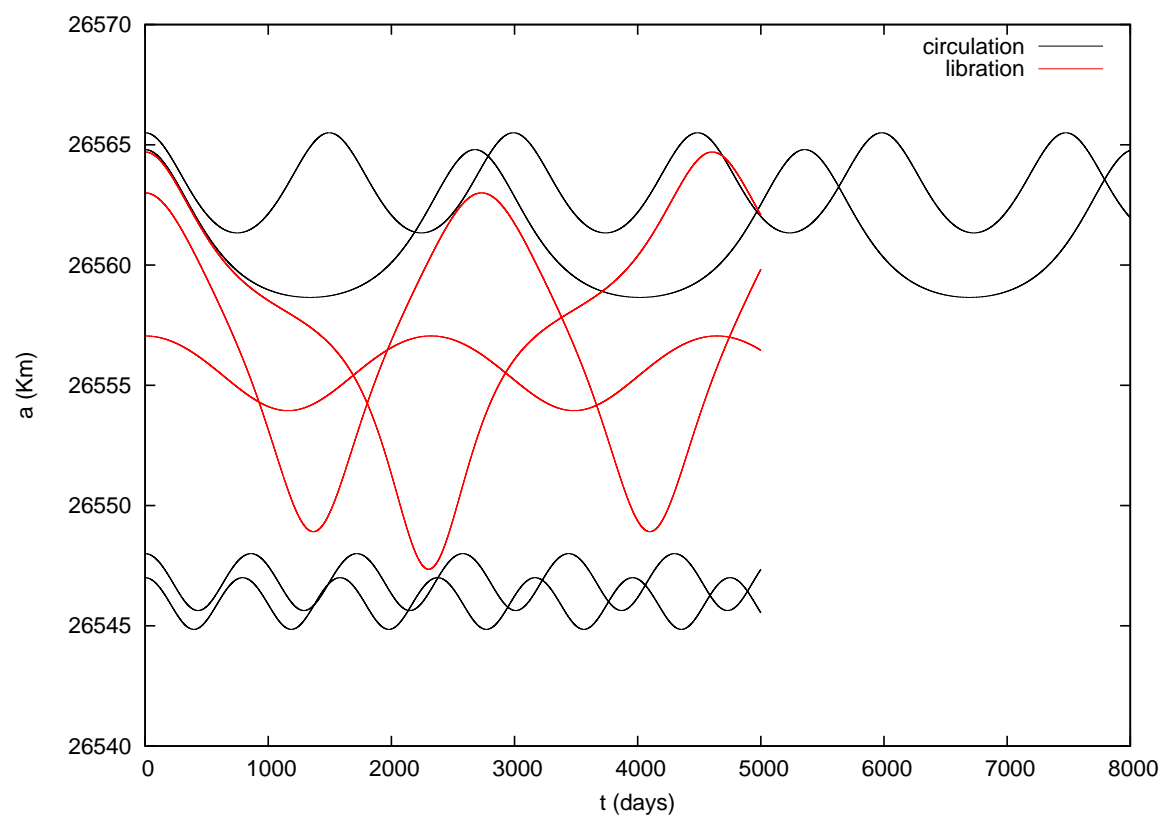

Figure 6. $a$ versus t, considering the tesseral harmonic $J_{22}$. The initial conditions for inclination and eccentricity are $I=10^{\circ}$ and $\mathrm{e}=0.01$, respectively 


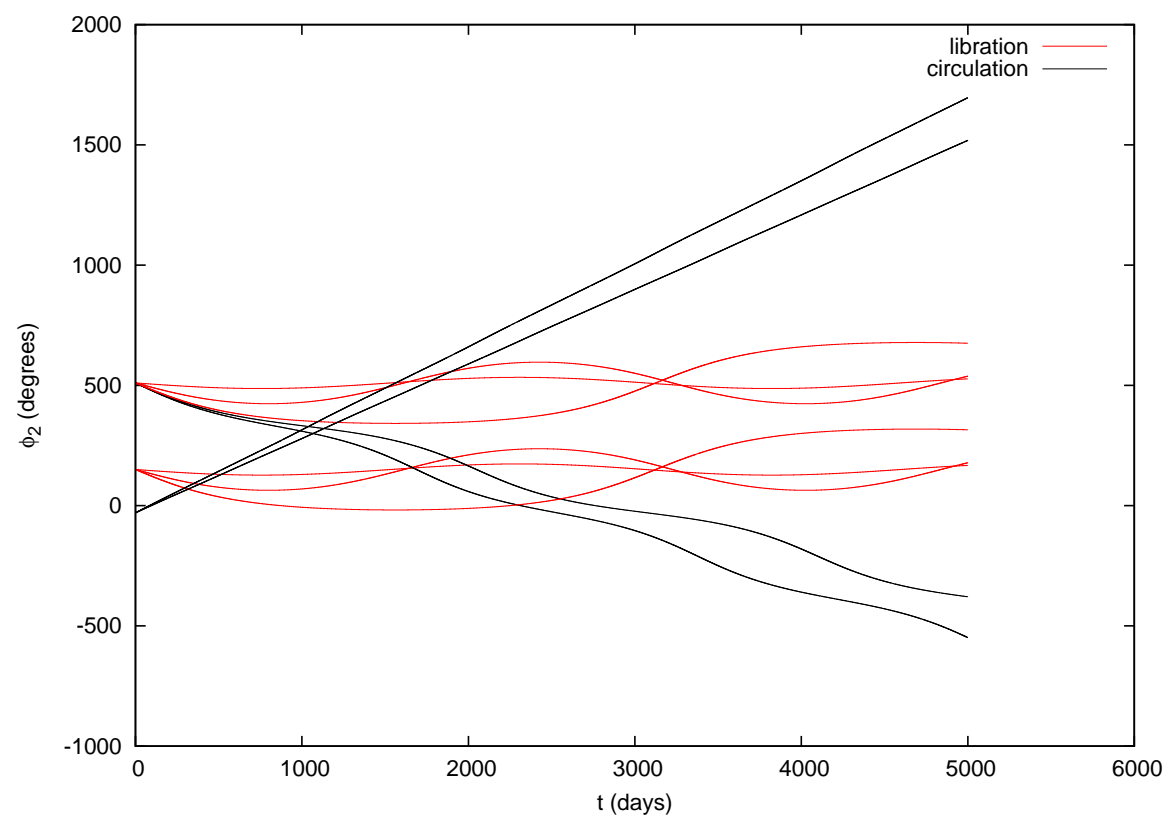

Figure 7. $\phi_{2}$ versus t, considering the tesseral harmonic $J_{22}$. The initial conditions for inclination and eccentricity are $I=55^{\circ}$ and $\mathrm{e}=0.01$, respectively

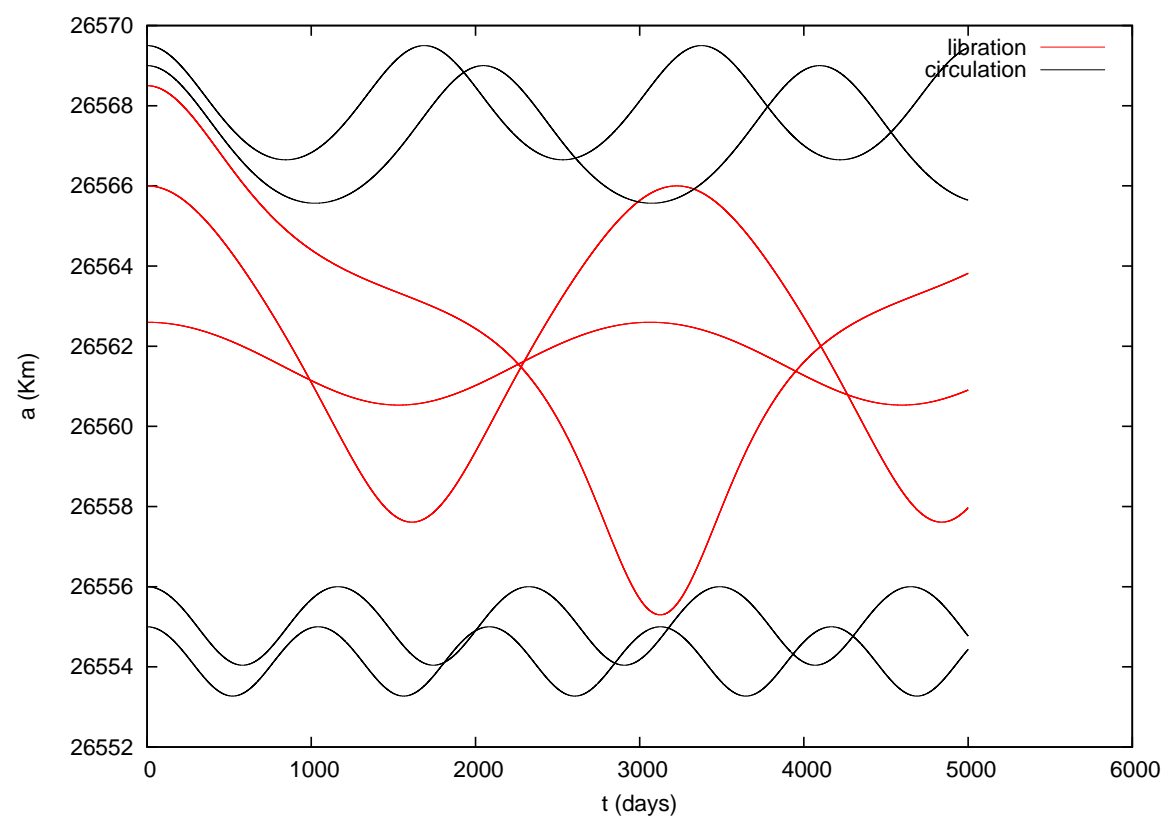

Figure 8. $a$ versus t, considering the tesseral harmonic $J_{22}$. The initial conditions for inclination and eccentricity are $I=55^{\circ}$ and $\mathrm{e}=0.01$, respectively 
Figures (1) to (8) show that vibration amplitudes increasing when the inclination varies from $10^{\circ}$ to $55^{\circ}$ and when the eccentricity varies from 0.001 to 0.01 . Figures (9) and (10) show the time behavior of the semi-major axis and $\phi_{2}$ angle, considering the critical angle $\phi_{2201}$ associated to tesseral harmonic $J_{22}$ and the critical angle $\phi_{4211}$ associated to tesseral harmonic $J_{42}$. The Table 1 show the resonant coefficients.

Table 1. Resonant coefficients

\begin{tabular}{|c|c|c|c|}
\hline Degree $(l)$ & Order $(m)$ & $p$ & $q$ \\
\hline 2 & 2 & 0 & 1 \\
\hline 4 & 2 & 1 & 1 \\
\hline
\end{tabular}

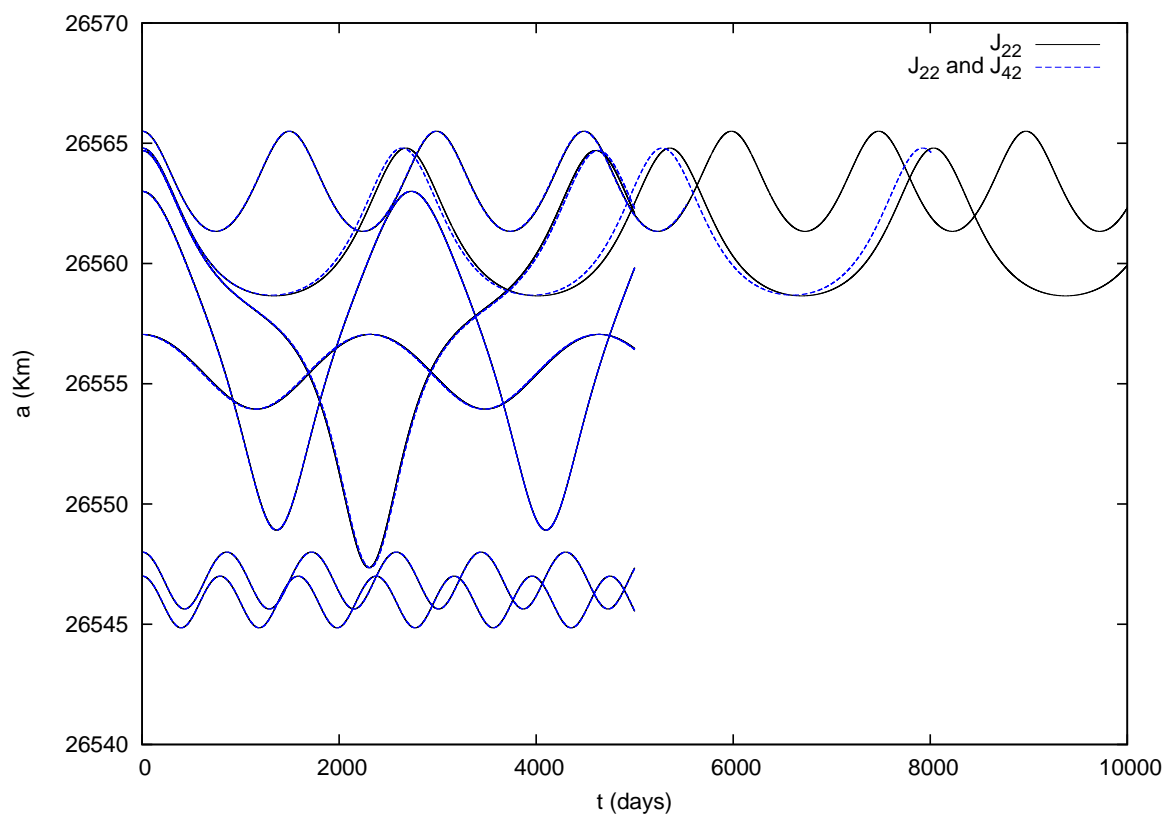

Figure 9. $a$ versus t, considering the tesseral harmonics $J_{22}$ and $J_{42}$. The initial conditions for inclination and eccentricity are $I=10^{\circ}$ and $\mathrm{e}=0.01$, respectively

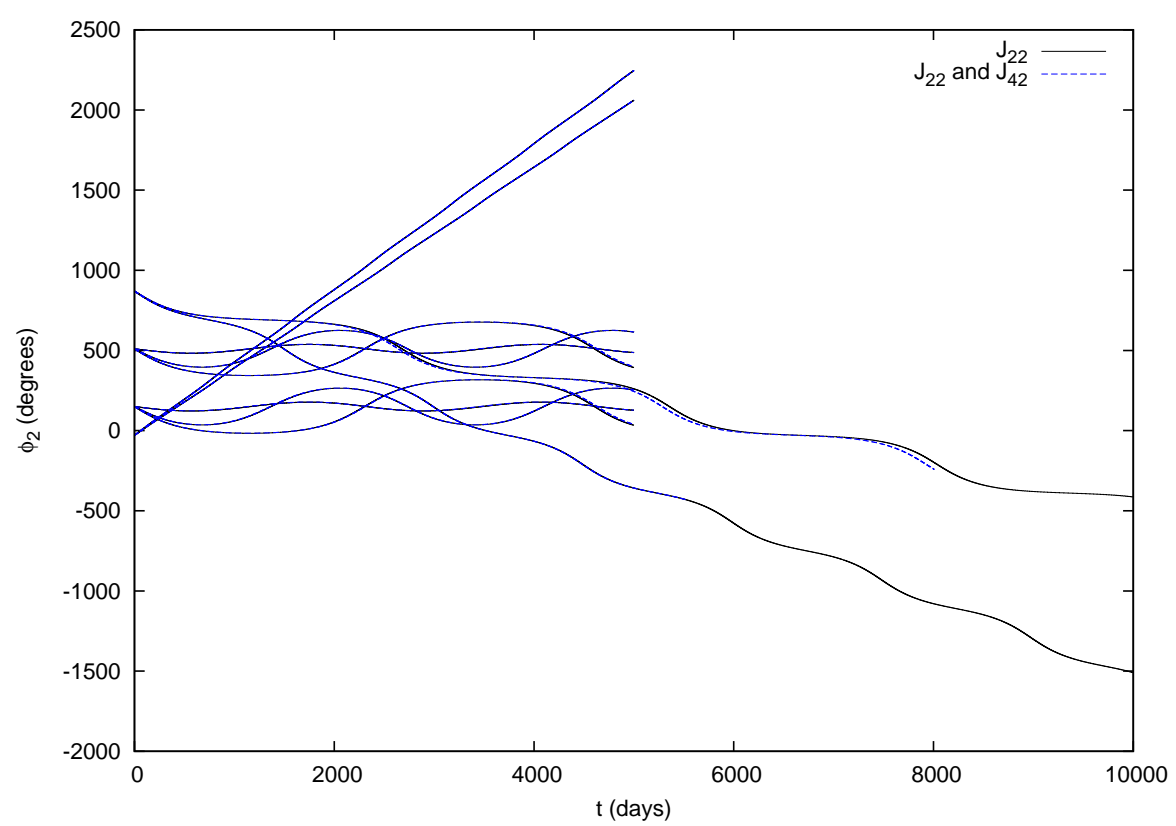

Figure 10. $\phi_{2}$ versus t, considering the tesseral harmonic $J_{22}$ and $J_{42}$. The initial conditions for inclination and eccentricity are $I=10^{\circ}$ and $\mathrm{e}=0.01$, respectively

Figures (9) and (10) show the difference in the time behavior of the semi-major axis and $\phi_{2}$ angle, with the addition of the tesseral harmonic $J_{42}$. The tesseral harmonic $J_{42}$ is $\cong O\left(10^{-6}\right)$ and it changes the value of the critical semimajor axis, in some centimeters, see the Tab. (2). The Tab. (2) show the critical semi-major axis for different 
inclinations and eccentricities and it shows the difference in the numerical value of the semi-major axis, when the tesseral harmonic $J_{42}$ is included. These critical values are obtained from the following condition

$$
\frac{\mu^{2}}{X_{2}^{3}}-2 \omega_{e}-\sum_{j=1}^{\infty} \frac{\partial B_{2,2 j, 0, j, 0}\left(X_{2}, C_{1}, C_{2}\right)}{\partial X_{2}}=0
$$

Table 2. Critical semi-major axis

\begin{tabular}{|c|c|c|c|}
\hline Eccentricity & Inclination $\left(^{\circ}\right)$ & Critical angle & Semi-major axis $(\mathrm{km})$ \\
\hline 0.001 & 10 & $\phi_{2201}$ & 26557.05255 \\
\hline 0.001 & 55 & $\phi_{2201}$ & 26562.59742 \\
\hline 0.01 & 10 & $\phi_{2201}$ & 26557.05208 \\
\hline 0.01 & 55 & $\phi_{2201}$ & 26562.59758 \\
\hline 0.001 & 10 & $\phi_{2201}+\phi_{4211}$ & 26557.05194 \\
\hline 0.001 & 55 & $\phi_{2201}+\phi_{4211}$ & 26562.59767 \\
\hline 0.01 & 10 & $\phi_{2201}+\phi_{4211}$ & 26557.05148 \\
\hline 0.01 & 55 & $\phi_{2201}+\phi_{4211}$ & 26562.59783 \\
\hline
\end{tabular}

\section{Conclusions}

In this work, the dynamical behavior of two critical angles associated to the $2: 1$ resonance problem in the artificial satellites motion have been investigated. Through successive canonical transformations, a simplified model describing the problem is derived. In the regular motion region, one can study the dynamical system considering each critical angle separately.

The results show the time behavior of the semi-major axis and $\phi_{2}$ angle, considering two inclinations, $10^{0}$ and $55^{0}$, and different eccentricities, 0.001 and 0.01 . Two different regions are observed in the numerical integration, libration and circulation regions.

Two critical angles are studied, $\phi_{2201}$ associated to $J_{22}$ and $\phi_{4211}$ associated to $J_{42}$. The values of the critical semi-major axis show a difference of centimeters in the libration region, when the tesseral harmonic $J_{42}$ is added.

Inside the region where the resonances are found, the motion can be chaotic, because it shows sensibility to initial conditions.

\section{Acknowledgements}

This work was accomplished with support of the FAPESP under the contract $\mathrm{N}^{o}$ 2009/00735-5 and 2006/04997-6, SP-Brazil, and CNPQ (contracts 300952/2008-2 and 302949/2009-7).

\section{References}

Morando, M. B. Orbites de Resonance des Satellites de 24h. Bull. Astron. 24, pp. 47, 1963.

Blitzer, L. Synchronous and Resonant Satellite Orbits Associated with Equatorial Ellipticity. ARS Journal 32, pp. 1016-1019, 1963.

Garfinkel, B. The Disturbing Function for an Artificial Satellite. Astron. Journal, Vol. 70, pp. 688-704, 1965a.

Garfinkel, B. Tesseral Harmonic Perturbations of an Artificial Satellite. Astron. Journal, Vol. 70, pp. 784-786, 1965b.

Garfinkel, B. Formal Solution in the Problem of Small Divisors. Astron. Journal, Vol. 71, pp. 657-669, 1966.

Gedeon, G. S; Dial, O. L. Along-track Oscillations of a Satellite due to Tesseral Harmonics. AIAA Journal, Vol. 5, pp. 593-595, 1967.

Gedeon, G. S; Douglas, B. C; Palmiter, M. T. Resonance Effects on Eccentric Satellite Orbits. Journal of the Astronautical Sciences, Vol. XIV, pp. 147-157, 1967.

Gedeon, G. S. Tesseral Resonance Effects on Satellite Orbits. Celestial Mechanics 1, pp. 167-189, 1969.

Lane, M. T. An Analytical Treatment of Resonance Effects on Satellite Orbits. Celestial Mechanics, 42, pp. 3-38, 1988. 
Jupp, A. A Solution of the Ideal Resonance Problem for the Case of Libration. Astron. Journal, Vol. 74, pp. 35-43, 1969.

Ely, T. A; Howell, K. C. Long-term Evolution of Artificial Satellite Orbits due to Resonant Tesseral Harmonics. Journal of the Astronautical Sciences, Vol. 44, pp. 167-190, 1996.

Neto, A. G. S.. Estudo de Órbitas Ressonantes no Movimento de Satélites Artificiais. Tese de Mestrado, ITA, 2006.

Osorio, J. P.. Perturbações de Órbitas de Satélites no Estudo do Campo Gravitacional Terrestre. Porto, Imprensa Portuguesa, 1973.

Kaula, W. M. Theory of Satellite Geodesy:Applications of Satellites to Geodesy. Blaisdel Publ. Co., Waltham, Mass, 1966.

Lima Jr, P. H. C. N. Sistemas Ressonantes a Altas Excentricidades no Movimento de Satélites Artificiais. Tese de Doutorado, Instituto Tecnológico de Aeronáutica, 1998.

Grosso, P. R. Movimento Orbital de um Satélite Artificial em Ressonância 2:1. Tese de Mestrado, Instituto Tecnológico de Aeronáutica, 1989.

Formiga, J. K. S.; Vilhena de Moraes, R. Dynamical systems: an integrable kernel for resonance effects. Journal of Computational Interdisciplinary Sciences 1(2): pp. 89-94, 2009.

Vilhena de Moraes, R.; Fitzgibbon, K. T.; Konemba, M. Influence of the 2:1 Resonance in the Orbits of GPS Satellites. Adv. Space Res., COSPAR, Vol. 16, No 12, pp. 37-40, Grã Bretanha, 1995. 